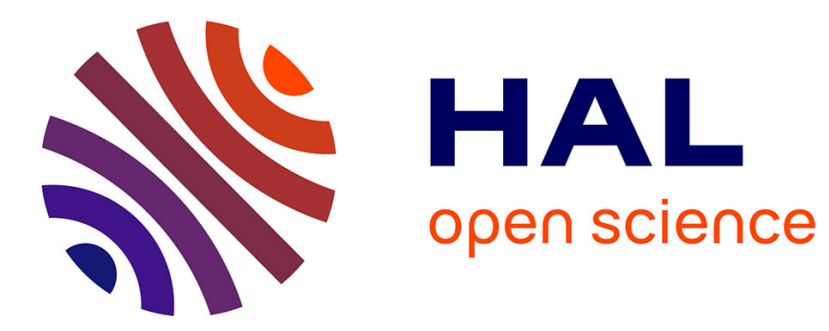

\title{
Computational Challenges of Nonlinear Systems
}

Laurette Tuckerman

\section{To cite this version:}

Laurette Tuckerman. Computational Challenges of Nonlinear Systems. Emerging Frontiers in Nonlinear Science, pp.249-277, 2020, 10.1007/978-3-030-44992-6_11 . hal-02995001

\section{HAL Id: hal-02995001 \\ https://hal.science/hal-02995001}

Submitted on 5 Jan 2021

HAL is a multi-disciplinary open access archive for the deposit and dissemination of scientific research documents, whether they are published or not. The documents may come from teaching and research institutions in France or abroad, or from public or private research centers.
L'archive ouverte pluridisciplinaire HAL, est destinée au dépôt et à la diffusion de documents scientifiques de niveau recherche, publiés ou non, émanant des établissements d'enseignement et de recherche français ou étrangers, des laboratoires publics ou privés. 


\title{
Computational Challenges of Nonlinear Systems
}

Laurette S. Tuckerman

\begin{abstract}
We survey some of the major types of dynamical-systems computations that can be carried out for two or three-dimensional systems of partial differential equations. In order of increasing complexity, we describe methods for calculating steady states and bifurcation diagrams, linear stability and Floquet analysis, and heteroclinic orbits. These are illustrated by computations for Rayleigh-Bénard convection in a cylindrical geometry, the Faraday instability of a fluid layer, the flow past a cylinder and over a square cavity, flow in a cylindrical container with counter-rotating lids, and Bose-Einstein condensation. We discuss some mathematical questions raised by these computations and the need for improved numerical tools.
\end{abstract}

\section{Time integration}

Many dynamical systems of interest in the sciences and engineering can be written as

$$
\partial_{t} U=\mathcal{F}(U)=\mathcal{L} U+\mathcal{N}(U)
$$

where $U$ consists of one or more functions of spatial variables $x,(x, y)$ or $(x, y, z)$, and $\mathcal{F}$ combines algebraic and differential operators. In (1), the evolution operator $\mathcal{F}$ has been decomposed into a linear operator $\mathcal{L}$, while $\mathcal{N}$ contains any other terms. Examples of such dynamical systems are the Navier-Stokes equations, the Boussinesq equations, the Swift-Hohenberg equation, and the Nonlinear Schrödinger equation. $\mathcal{L}$ is often the Laplacian $\nabla^{2}$ but may also be a higher-order differential operator, as in

Laurette S. Tuckerman

Laboratoire de Physique et Mécanique des Milieux Hétérogènes (PMMH),

CNRS, ESPCI Paris, PSL Research University;

Sorbonne Université, Univ. Paris Diderot,

75005 France

e-mail: laurette@pmmh.espci.fr 
the case of the Swift-Hohenberg equation, and it may contain other types of terms, such as buoyancy forces.

The most well-known and fundamental role of computation in nonlinear dynamics is that of a perfect experiment: following the evolution of $U(t)$ in time from an initial condition $U_{0}$. Geometries and forces can be implemented perfectly, without noise, and parameters can be scanned, even taking on values that are not physically realizable. In addition, numerical solutions provide all of the details of the system, e.g. the velocity or temperature at every point in space. Research communities in computational physics, chemistry, and engineering all have their own preferred methods for discretizing partial differential equations in space and time.

Numerical integration in time can be seen as replacing the temporally continuous dynamical system (1) by a temporally discrete system. The best choice of method is dictated by the nature of $\mathcal{F}$. If (1) is a Hamiltonian system, then methods which conserve volume in phase space are essential. If $\mathcal{L}$ represents a highly dissipative operator, then implicit methods are required for its integration. This is a vast topic and here we will consider only the simple first-order Euler implicit-explicit scheme:

leading to

$$
U(t+\Delta t)=U(t)+\Delta t(\mathcal{L} U(t+\Delta t)+\mathcal{N}(U(t)))
$$

$$
U(t+\Delta t)=\mathcal{B}_{\Delta t} U(t) \equiv(I-\Delta t \mathcal{L})^{-1}(I+\Delta t \mathcal{N}) U(t)
$$

Scheme (2) will be used as a building block for other algorithms. When the goal is time integration, other schemes that are more accurate than (2) can be used.

\section{Steady states and bifurcation diagrams: cylindrical convection}

Dynamical systems can be organized around objects of increasing complexity: steady states, periodic orbits, and then tori, heteroclinic orbits, and so on. The study of the steady-state problem might seem prosaic, but in fact it is anything but that. For motivation, we present in Figs. 1-8 the results of experimental and numerical investigations of Rayleigh-Bénard convection in a fluid-filled cylinder whose radius is twice the height and whose upper and lower boundaries are maintained at different temperatures.

The governing equations of this system are the Navier-Stokes and Boussinesq equations:

$$
\begin{aligned}
\partial_{t} H+(\mathbf{U} \cdot \nabla) H & =R a U_{z}+\nabla^{2} H \\
\operatorname{Pr}^{-1}\left(\partial_{t} \mathbf{U}+(\mathbf{U} \cdot \nabla) \mathbf{U}\right) & =-\nabla P+\nabla^{2} \mathbf{U}+H \mathbf{e}_{z} \\
\nabla \cdot \mathbf{U} & =0
\end{aligned}
$$

The conductive temperature profiles is linear in the vertical direction; $H$ is the deviation of the temperature from this profile while $\mathbf{U}$ is the velocity and $P$ is 
the pressure. $R a$ is the Rayleigh number, which is a nondimensional measure of the temperature difference imposed between the upper and lower plates, and $P r$ is the Prandtl number, which is the ratio of the kinematic viscosity to the thermal diffusivities. The boundary conditions for the velocity correspond to no-penetration and no-slip on the horizontal plates and sidewalls:

$$
\mathbf{U}=0 \quad \text { for } \quad z= \pm 1 / 2 \text { and for } \quad r=2 .
$$

The boundary conditions on the temperature correspond to perfectly conducting horizontal bounding plates and perfectly insulating sidewalls:

$$
H=0 \text { for } z= \pm 1 / 2 \text { and } \partial_{r} H=0 \text { for } r=2
$$

In their experiments, Hof et al. [1] observed a multiplicity of distinct patterns - tori, dipoles, roll patterns squeezed into a baseball-like shape, and a three-fold pattern that they named mercedes - at the same Rayleigh and Prandtl numbers, as shown in Fig. 1. Boronska \& Tuckerman were able to reproduce these states [2] and found additional states as well, shown in Fig. 2, by numerical time integration via, e.g. (2), of the spatially discretized equations (3a)-(4b) in a domain with resolution $N_{r} \times N_{\theta} \times N_{z}=40 \times 120 \times 20 \approx 10^{5}$, i.e. state vectors of size $M \approx 4 \times 10^{5}$.

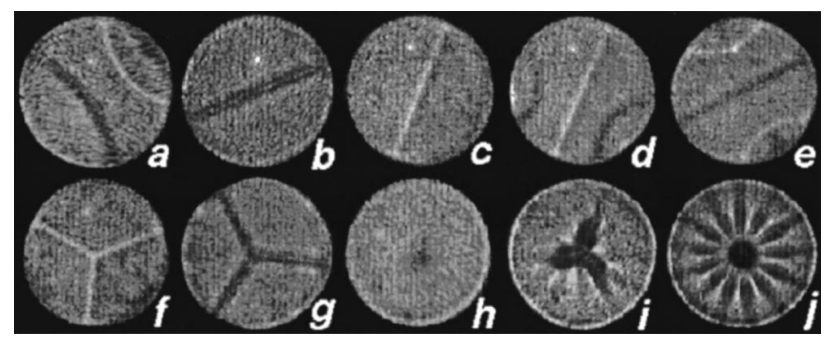

Fig. 1 Patterns observed in the experiment of Hof et al.. (a)-(h) are all observed at $R a=14200$. $(a)$ three rolls, $(b)$ two rolls, $(c)$ inverted two rolls, $(d)$ four rolls, $(e)$ inverted four rolls, $(f)$ mercedes, $(g)$ inverted mercedes, $(h)$ axisymmetric pattern. (i) is a rotating pattern at $R a=26600$ while (j) is a pulsed spoked pattern at $R a=33000$. Dark areas correspond to hot (rising) and bright to cold (descending) fluid. Reproduced from [1]. (C1999 by the American Institute of Physics

An understanding of the origin of these states and their connections and ranges of existence and stability can only be obtained from a full bifurcation-theoretic study. This was done by Boronska \& Tuckerman [3] in a large-scale calculation via methods which we will describe below. The bifurcation diagram which we have computed is shown in Fig. 3. As complicated as it appears, it is still incomplete: many other branches exist which have not been followed and so have been omitted.

Below the convective threshold, the system has symmetry $O(2) \times Z_{2}$, derived from the azimuthal rotation and reflection symmetry of the cylinder, and the Boussinesq reflection symmetry. Basic principles of bifurcation theory then dictate that branches 


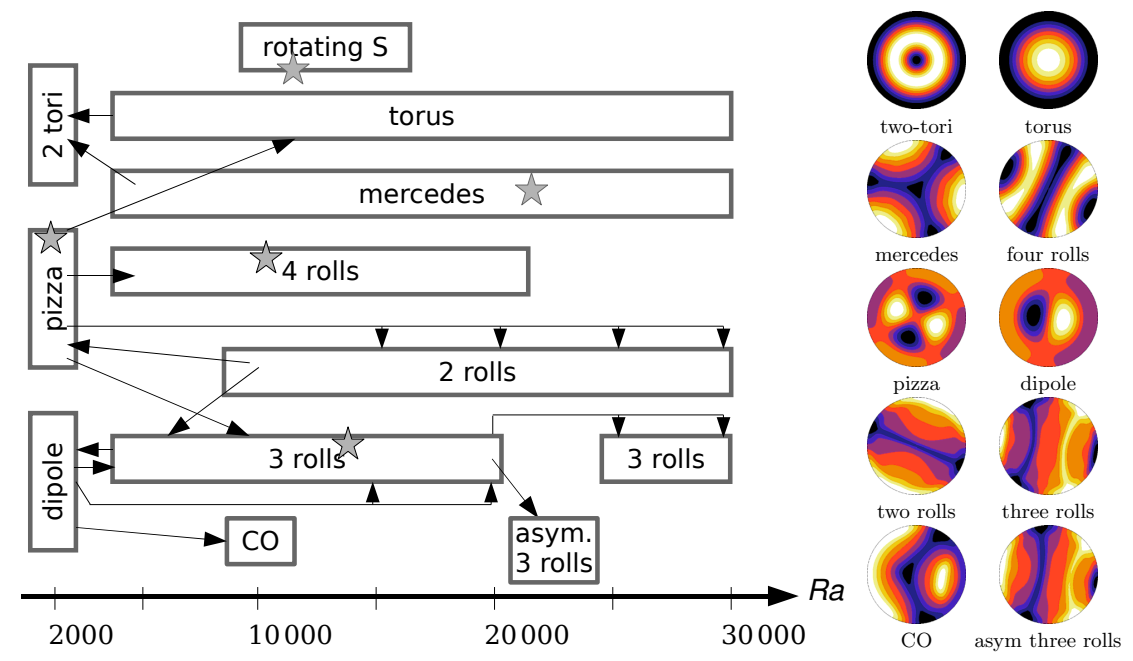

Fig. 2 Overview of the patterns observed in time-dependent simulations of convection in a cylinder with insulating radial wall. Initial simulations use a small perturbation of the conductive state at the Rayleigh numbers and lead to the patterns indicated by stars. The Rayleigh number was then raised or lowered, either gradually, or abruptly as indicated by the arrows. The panels on the right show the temperature field in the midplane, with rising warm fluid indicated by light colors and descending cold fluid by dark colors. Reproduced from [3]

bifurcating from the conductive state must have a trigonometric dependence on $\theta$ with some azimuthal wavenumber $m$, and Fig. 4 shows that this is indeed the case.

In Figs. 5 and 6, we follow the branches emanating from the $m=0$ (axisymmetric) and $m=3$ bifurcations. Figure 5 shows surprising and specific features: the branch which bifurcates from the conductive state disappears again after undergoing a saddle-node bifurcation, and a second disconnected set of axisymmetric states exists. Figure 6 shows the tortuous path taken by the $m=3$ branch, from its birth at a bifurcation from the conductive state (breaking the $O(2)$ symmetry) followed by a second pitchfork bifurcation (breaking the remaining $Z_{2}$ symmetry) and then two saddle-node bifurcations, finally leading to the mercedes states.

We now explain the computational method by which the bifurcation diagrams in Figs. 3-6 have been calculated. Steady states are solutions to

$$
0=\mathcal{F}(U)
$$

The main way to find steady states, i.e. to solve (5), is via Newton's method

$$
\begin{aligned}
\mathcal{F}_{U} u & =\mathcal{F}(U) \\
U & \leftarrow U-u
\end{aligned}
$$




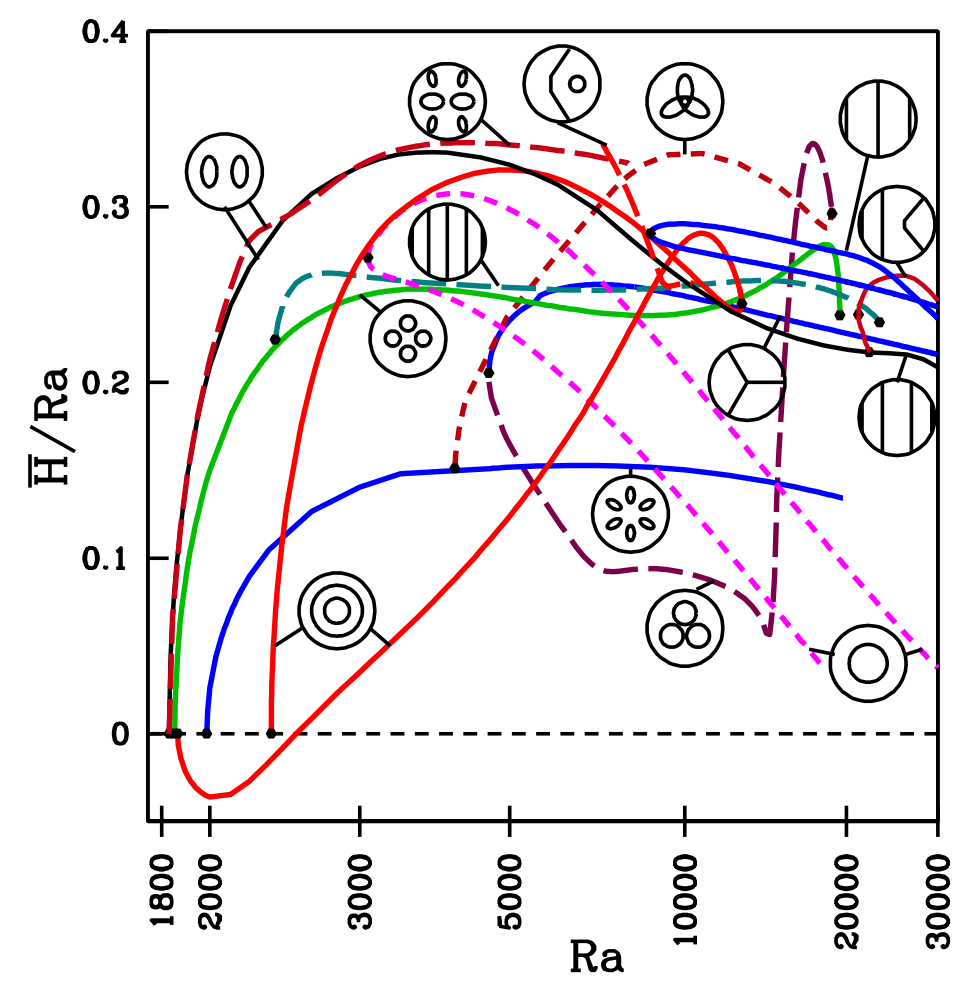

Fig. 3 Bifurcation diagram for cylindrical convection. There are 17 branches of steady states, as well as the conductive branch (shown as the short-dashed horizontal line). We call the branches pizza (solid green), four-roll (long-dashed turquoise), two-tori (solid red; 2), torus (long-dashed magenta; 2), marigold (solid blue), mitsubishi (short-dashed purple), cloverleaf (long-dashed purple) and mercedes (solid blue), three-roll (solid black), tiger (long-dashed brick), asymmetric three-roll (solid brick; 2), two-roll (solid blue; 2), and CO (long-dashed red), where $(\cdot ; 2)$ indicates related pairs of branches. Dots indicate turning points or pitchfork bifurcations. No information about stability is provided in this diagram. Reproduced from [3]

where the subscript $U$ designates, here and elsewhere, the linearization of an operator about $U$. The main challenge is the solution of the linear system (6). Indeed, calculating the solutions of linear systems and the eigenvectors of large matrices is the main bottleneck in numerical computations for studying dynamical systems.

Combining (1) and (6), Newton's method calls for solving the linear system

$$
\left(\mathcal{L}+\mathcal{N}_{U}\right) u=(\mathcal{L}+\mathcal{N}) U
$$

where $\mathcal{N}_{U}$ is the linearization of $\mathcal{N}$ about the steady state $U$. Linear systems can be solved directly or iteratively. For the calculations shown in Figs. 5 and 6, the domain 
Fig. 4 Primary bifurcations from conductive state. The first four critical wavenumber and Rayleigh numbers are ( $m=1, R a=1828$; black), ( $m=2, R a=1849 ;$ green $),$ ( $m=0, R a=1861$; red), and ( $m=3, R a=1985$; blue) for the radius-to-height aspect ratio or 2 with insulating radial walls. Reproduced from [3]

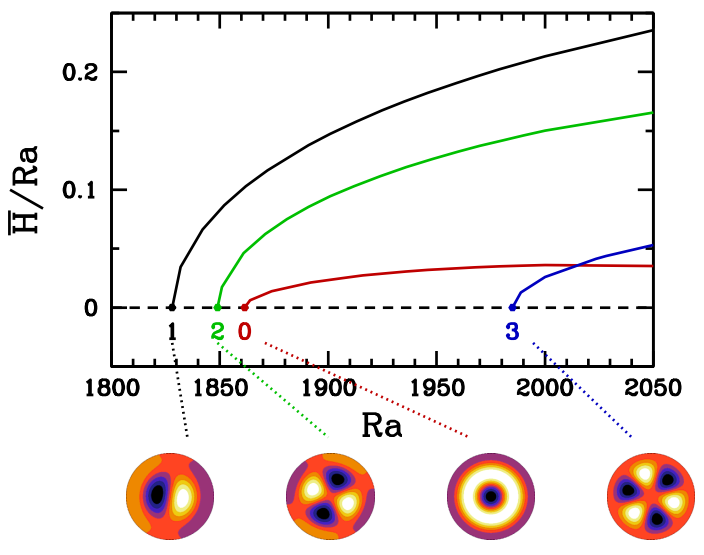

is represented by nearly $10^{5}$ points and the state vector is of size $M=4 \times 10^{5}$. Inverting a matrix of this size directly is usually out of the question for a discretized PDE in two or three spatial dimensions, since inversion takes a time proportional to $M^{3}$.

The class of methods called Krylov methods generalize the conjugate gradient (CG) method to matrices which are not symmetric definite and include the algorithms GMRES [4], BiCGSTAB [5] and IDR [6]; interested readers should consult these references. These methods construct a solution from the Krylov vectors, formed by taking successive products of the matrix and the right-hand-side vector. In order to simplify the notation, we write the problem we wish to solve as

$$
A x=b
$$

The general idea is to act repeatedly with $A$ in order to form vectors

$$
b, A b, A^{2} b, \ldots A^{K-1} b
$$

These vectors - or orthonormalized versions of them - are called Krylov vectors and their linear combinations comprise the Krylov space. Krylov methods approximate the solution $x$ within the Krylov space:

$$
x \approx \sum_{k=0}^{K-1} c_{k} A^{k} b
$$

In the worst-case analysis, if the number $K$ of Krylov vectors attains the size $M$ of the vectors, then generically the Krylov space spans $\mathcal{R}^{M}$ and $x$ can always be expressed via (10). Since a general matrix-vector product requires $O\left(M^{2}\right)$ operations, the operation count for constructing $M$ Krylov vectors is $O\left(M^{3}\right)$, the same as for a direct method. 


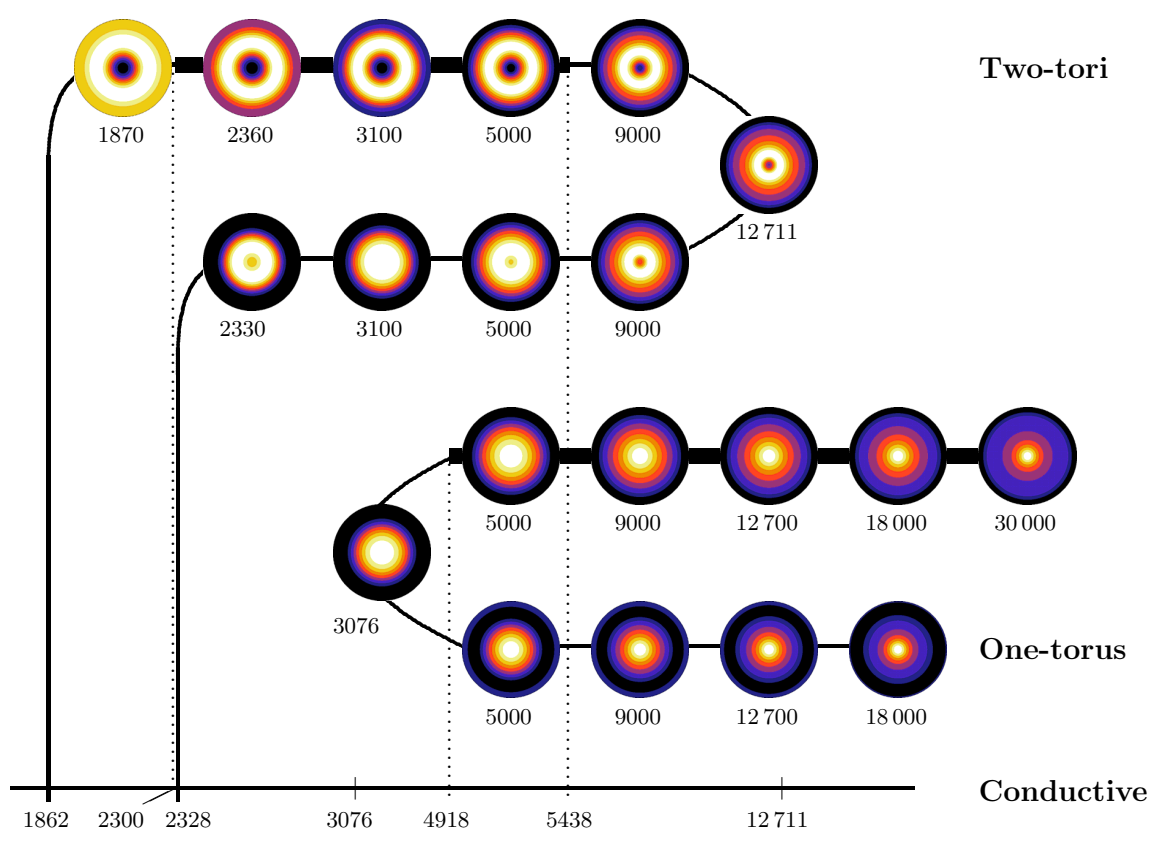

Fig. 5 Axisymmetric branches displayed via a schematic partial bifurcation diagram. Pitchfork bifurcations at $R a=1862$ and 2328 from the conductive state lead to the two-tori branches, which are themselves connected via a saddle-node bifurcation at $R a=12711$; the upper one is stable for $2300 \leq R a \leq 5438$. A turning point at $R a=3076$ leads to the one-torus branches; the upper one is stable for $R a \geq 4918$. Reproduced from [3]

The challenge is thus to develop algorithms which are less time-consuming. A first savings is realized from the fact that matrices derived from spatially discretizating PDEs are not arbitrary: the time required to act with them (rather than inverting them) is typically closer to $M$ than to $M^{2}$. A second economy is realized by reducing $K \ll M$. The number $K$ of Krylov vectors required to approximate the solution depends on the condition number of the matrix, basically the ratio of its largest to its smallest eigenvalue. By preconditioning, i.e. by acting on both sides of (8) with an operator which approximates the inverse of $A$, the ratio between maximum and minimum eigenvalues is reduced. The matrix in (7) is badly conditioned, primarily (in the cases of interest to us) because $\mathcal{L}$ is. We multiply (7) by $(I-T \mathcal{L})^{-1} T$, where $T$ is a large timestep. This acts to counterbalance $\mathcal{L}$ and leads to

$$
\begin{aligned}
(I-T \mathcal{L})^{-1} T\left(\mathcal{L}+\mathcal{N}_{U}\right) u & =(I-T \mathcal{L})^{-1} T(\mathcal{L}+\mathcal{N}) U \\
(I-T \mathcal{L})^{-1}\left(I+T \mathcal{N}_{U}-I+T \mathcal{L}\right) u & =(I-T \mathcal{L})^{-1}(I+T \mathcal{N}-I+T \mathcal{L}) U \\
{\left[(I-T \mathcal{L})^{-1}\left(I+T \mathcal{N}_{U}\right)-I\right] u } & =\left[(I-T \mathcal{L})^{-1}(I+T \mathcal{N})-I\right] U \\
{\left[\mathcal{B}_{U, T}-I\right] u } & =\left[\mathcal{B}_{T}-I\right] U
\end{aligned}
$$




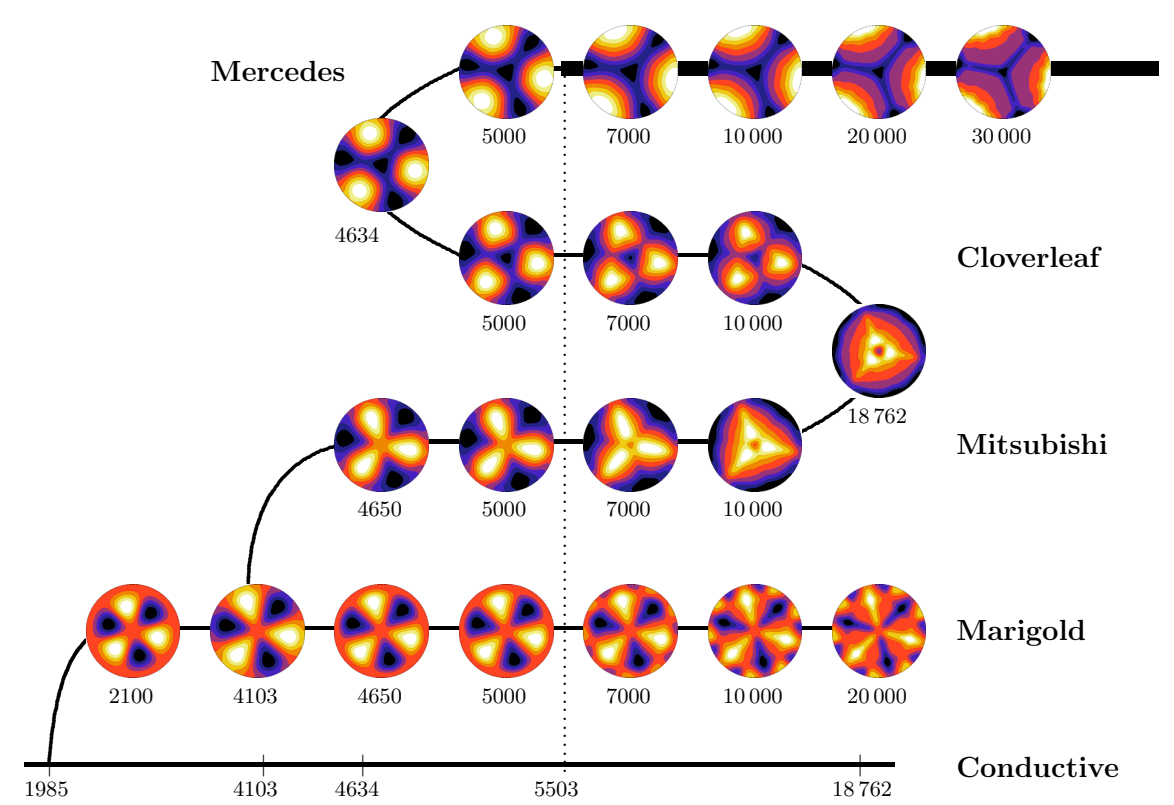

Fig. 6 Branches originating from the $m=3$ bifurcation displayed via a schematic partial bifurcation diagram. The Marigold branch is created via an $m=3$ circle pitchfork bifurcation from the Conductive branch at $R a=1985$. A pitchfork bifurcation at $R a=4103$, creates the Mitsubishi branch. A saddle-node at $R a=18762$ produces the Cloverleaf branch, and another saddle-node at $R a=4634$ leads to the Mercedes branch. The thick line for $R a>5503$ indicates the only stable regime, a portion of the Mercedes branch. Reproduced from [3]

where $\mathcal{B}_{U, T} \equiv(I-T \mathcal{L})^{-1}\left(I+T \mathcal{N}_{U}\right)$. (We will omit subscripts when they seem unnecessary.) Equation (11) shows that steady states, which are the roots of $(\mathcal{L}+\mathcal{N})$, are also roots of the operator $\left(\mathcal{B}_{T}-I\right)$, where $\mathcal{B}_{T}$ is obtained from a single Euler timestep (2), for any value of $T$. A large value of $T$, on the order of $T=100$ or 1000 , serves to insure that $(I-T \mathcal{L})^{-1} T \approx-\mathcal{L}^{-1}$. Solving (11) using method BiCGSTAB for the cylindrical convection problem above then requires a much smaller (30-800) number of Krylov vectors $K$ than the system size $M=4 \times 10^{5}$. This method, called Stokes preconditioning (because $\mathcal{L}$ is associated with the Stokes problem in hydrodynamics), has been presented and used in, e.g. [7, 8, 9, 10, 11, 12, 13, 14, 15].

Another method for finding steady states also relies on time-integration, using the operator $\mathcal{B}_{\Delta t}^{T / \Delta t}-I$, where $\Delta t$ is a timestep of conventional size, e.g. $\Delta t \approx 0.01$ for a typical non-dimensionalized hydrodynamic stability problem. Since for $\Delta t \ll 1$,

$$
\begin{aligned}
U(\Delta t) & \approx B_{\Delta t} U(0) \Longrightarrow U(T) \approx\left(B_{\Delta t}\right)^{T / \Delta t} U(0) \\
U(T)-U(0) & \approx\left(\left(B_{\Delta t}\right)^{T / \Delta t}-I\right) U(t)
\end{aligned}
$$


steady states are also roots of $\left(\left(B_{\Delta t}\right)^{T / \Delta t}-I\right)$ (in the limit $\left.\Delta t \rightarrow 0\right)$, and the corresponding Newton step is

$$
\left(\left(B_{U, \Delta t}\right)^{T / \Delta t}-I\right) u=\left(\left(B_{\Delta t}\right)^{T / \Delta t}-I\right) U
$$

We call this the integration method. Each operator action of (12) requires $T / \Delta t$ timesteps, rather than the single timestep required by the action of $\mathcal{B}_{T}-I$. However $\left(\left(B_{\Delta t}\right)^{T / \Delta t}-I\right)$ is better conditioned than $\mathcal{B}_{T}-I$ and so fewer Krylov vectors are required to represent the solution.

Theories depicting transitional turbulence as chaotic trajectories visiting unstable steady states and traveling waves [16] have inspired the calculation of a large number of such states, mostly by method (12), e.g. [17]. (In this context, unstable steady states are often called Exact Coherent Structures.) In [18] a comparison of methods (12) and (11) has been carried out for the computation of unstable steady states for plane Couette flow via Channelflow [19] and traveling waves for pipe flow via OpenPipeFlow [20]. We find that the tradeoff between fewer operator actions for $\left(B_{\Delta t}\right)^{T / \Delta t}-I$ vs. fewer timesteps for each operator action for $\mathcal{B}_{T}-I$ is such that the $\mathcal{B}_{T}-I$ method is 10 to 50 times faster in the Reynolds-number range that we have studied. However, some time-integration codes rely on $\Delta t$ being small (in particular, to impose incompressibility), making them difficult to incorporate into a single-timestep Newton's method.

Traveling waves can be calculated using the same methods as steady states. Writing $U=U(x-C t), U$ is a solution to

$$
\partial_{t} U=-C U=\mathcal{F}(U)
$$

i.e. $(U, C)$ is a solution to

$$
0=C U+\mathcal{F}(U)
$$

An additional condition on the phase must be imposed to compensate for the additional variable $C$. Although traveling waves can be computed using either (11) or (12), general periodic orbits cannot use (11), since the entire periodic orbit must be integrated. That is, one must find roots of $U(T)-U(0)$ and not of $\mathcal{F}(U)$ or $C U+\mathcal{F}(U)$.

\section{Linear stability analysis}

In the bifurcation diagrams presented in Figs. 5 and 6, thick lines denote sections of branches in which the steady states are stable meaning, in this case, linearly stable. Unlike states computed via time-integration, which are necessarily stable, those computed by Newton's method can also be unstable. How does one then determine their stability? The linear stability of a steady state is controlled by the eigenvalues $\lambda_{j}$ of $\mathcal{F}_{U}$. If none of the eigenvalues have positive real parts, then the steady state is 

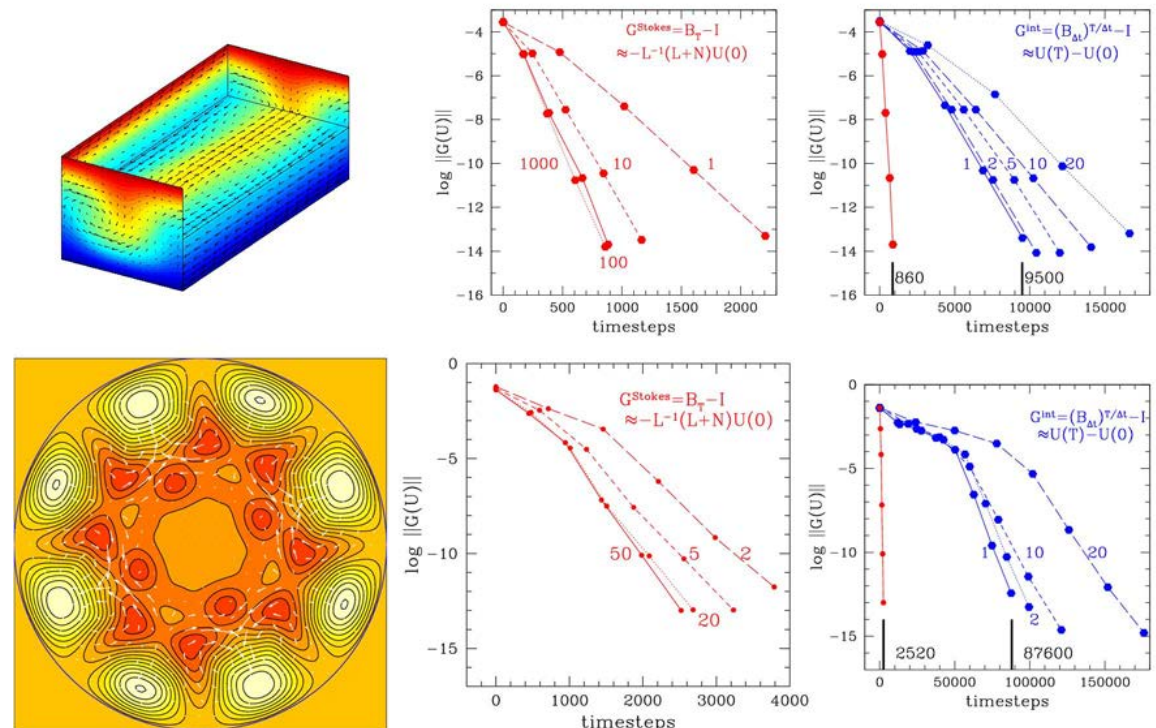

Fig. 7 Upper row: Steady state in plane Couette flow. Lower row: Traveling wave in pipe flow. Left column: depiction of flow. Middle and right columns: Performance of Newton's method, with timings measured by the number of timesteps taken. $U$ is considered to be a steady state when $\|G(U)\|<10^{-13}$. Dots show the number of timesteps at the end of each Newton step, while numbers show the time $T$ used in either a single large timestep or for multiple timesteps. Middle column: performance of Newton's method using single-timestep-method (11). Convergence is much faster when $T$ is increased, showing the effectiveness of Stokes preconditioning. Right column: performance of Newton's method using multiple-timestep-method (12). The single-timestep method is 11 times faster than the multiple-timestep method for the plane Couette flow calculation and 50 times faster for the pipe flow calculation. Top left panel is reproduced from [17] (C2009 by Cambridge University Press), bottom left panel is reproduced from [21] (@2008 by the Royal Society) and the rest of panels are reproduced from [18] (@2019 by Springer International Publishing)

stable. Otherwise it is unstable to perturbations of the form of unstable eigenvectors corresponding to the eigenvalues whose real parts are positive. In Fig. 8a, we show the leading eigenvalues corresponding to the axisymmetric states of Fig. 5. For the same reasons as for the conductive state, bifurcation-theoretic principles require that the corresponding eigenvectors each be trigonometric in the azimuthal direction, with a single wavenumber $m$. Another bifurcation-theoretic principle states that a new bifurcating branch (with azimuthal wavenumber $m$ ) is generated via a circle pitchfork at each zero-crossing of these eigenvalue curves. This is one of the reasons that many other branches exist in addition to those shown in Fig. 3, despite the complexity of this figure. Figure $8 \mathrm{~b}$ shows the single leading eigenvalue (that with largest real part) corresponding to the states in Fig. 6. States for which this eigenvalue is positive are unstable. From this figure it can be seen that all of the $m=3$ states are unstable until the leading eigenvalue of the mercedes branch finally becomes negative, as also seen in Fig. 6. 

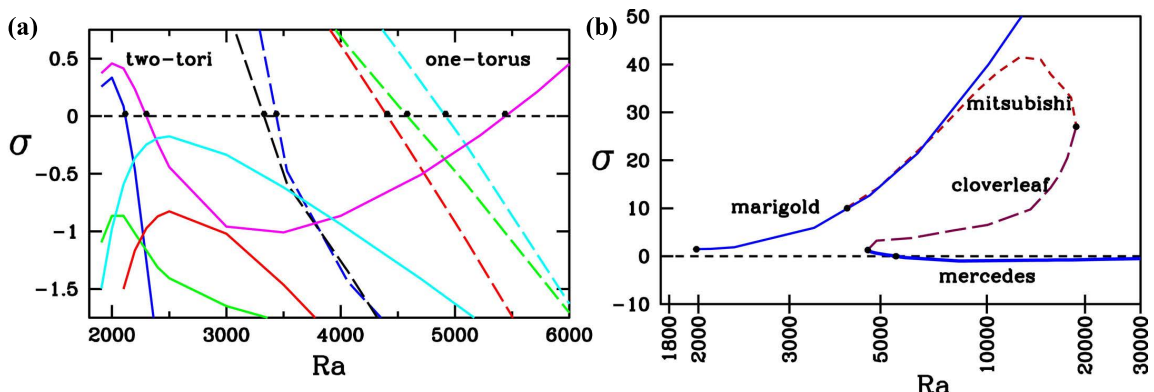

Fig. 8 (a) Leading eigenvalues of upper two-tori (solid) and one-torus (dashed) branches. The upper two-tori branch has two positive eigenvalues at onset, which subsequently cross zero, leading to stability over the interval $2300 \leq R a \leq 5438$. The upper one-torus branch has five positive eigenvalues at onset. These subsequently cross zero, leading to stability for $\operatorname{Re}>4918$, above which the branch is stable. Each zero-crossing is associated with the formation of a new branch. (b) Leading eigenvalue for each of the three-fold-symmetric branches: marigold, Mitsubishi, cloverleaf, Mercedes (rom highest to lowest). Dots indicate bifurcations between the branches and final stabilization of Mercedes branch for $R a>5503$. Reproduced from [3]

We now discuss the method by which these eigenvalues are computed. The most straightforward way to conduct linear stability analysis is to form the matrix $\mathcal{F}_{U}$ and to diagonalize it, i.e. to compute all of its eigenvalues. If, however, (1) consists of one or more partial differential equations in two or three spatial dimensions, then $U$ and $u$ are on the order of size $\left(\left(10^{2}\right)^{2}=10^{4}\right)$ or $\left(\left(10^{2}\right)^{3}=10^{6}\right)$, and so the matrix $\mathcal{F}_{U}$ is too large to diagonalize completely. The solution is to calculate only the leading eigenvalues, i.e. those whose real part is the largest and their eigenvectors. As for the steady state problem, we consider methods which build up a Krylov space by repeatedly applying an operator.

Like the Krylov methods for solving linear equations, those for finding selected eigenvalues rely on repeated operator action. The eigenvalues which are easiest to find are the dominant ones, those of largest magnitude. The simplest such method is the power method, in which only one vector is retained, which converges to the dominant eigenvector, that of largest magnitude. Generalizations, e.g. the block power method, converge to the dominant eigenvectors which, for dissipative systems like the Navier-Stokes equations, correspond to highly damped modes of $\mathcal{F}_{U}$ rather than the bifurcating modes which are of interest. We can apply a function to $\mathcal{F}_{U}$, using the fact that the eigenfunctions of a function of an operator are those of the original operator, while the eigenvalues are the function applied to the original eigenvalues. Thus, we continue to use the power method or the block power method, but on a different matrix whose dominant eigenvalues are those we seek.

Two such operator mappings are illustrated in Fig. 9. Consider the two operators

$$
\exp \left(\Delta t\left(\mathcal{L}+\mathcal{N}_{U}\right)\right) \quad \text { and } \quad\left(\mathcal{L}+\mathcal{N}_{U}\right)^{-1}
$$




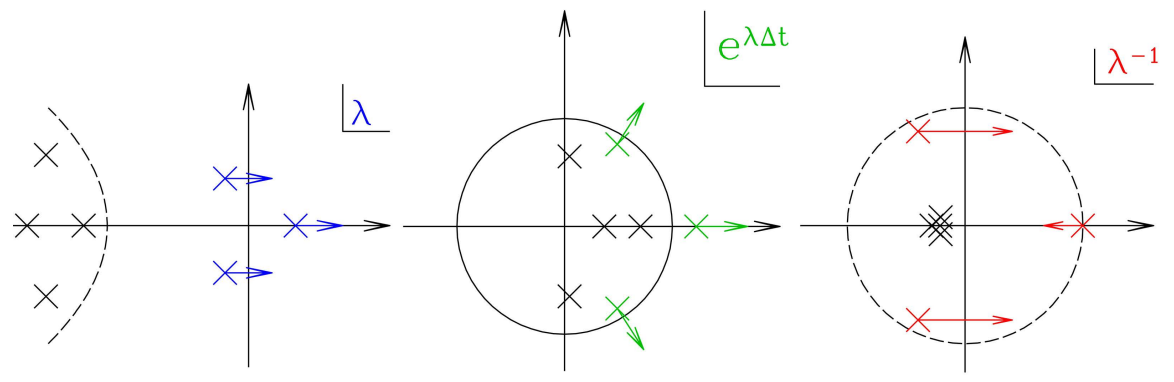

Fig. 9 Spectrum of a matrix A, along with that of $e^{A \Delta t}$ and $A^{-1}$. We seek the leading eigenvalues of $A$ (blue crosses) but these are difficult to obtain via the power or block power methods because they are not the dominant ones. When the exponential or inverse is applied to $A$, the leading eigenvalues of $A$ become the dominant ones of $e^{A \Delta t}$ (green crosses) and of $A^{-1}$ (red crosses) and are hence much easier to obtain.

We approximate the action of these operators on a vector $u^{(n)}$ by using variants of the Euler time-stepping operator (2). The exponential power method uses

$$
u^{(n+1)}=\mathcal{B}_{U, \Delta t} u^{(n)} \equiv(I-\Delta t \mathcal{L})^{-1}\left(I+\Delta t \mathcal{N}_{U}\right) u^{(n)} \approx \exp \left(\Delta t\left(\mathcal{L}+\mathcal{N}_{U}\right)\right) u^{(n)}
$$

for small $\Delta t$, while the inverse power method uses

$$
\left(\mathcal{L}+\mathcal{N}_{U}\right) u^{(n+1)}=u^{(n)}
$$

As seen in (11), equation (17) is equivalent to

$$
\left[(I-T \mathcal{L})^{-1}\left(I+T \mathcal{N}_{U}\right)-I\right] u^{(n+1)}=(I-T \mathcal{L})^{-1} u^{(n)}
$$

Equations (16) and (18) are analogous to the two methods described in (12) and (11) for computing steady states. For simplicity, we have presented the power method, which computes a single eigenpair. Both the exponential power method or the inverse power method can be generalized to compute a number of eigenpairs, by keeping a number $K$ of iterates of the matrix as follows. We renumber the first retained iterate to be $u_{0}$. The vectors $\left\{u_{0}, \ldots u_{K-1}\right\}$ are orthonormalized to yield a set $\left\{v_{0}, \ldots v_{K-1}\right\}$, whose span is the Krylov space. A $K \times K$ matrix is defined via $H_{i, j} \equiv\left\langle v_{i}, A v_{j}\right\rangle$. The small matrix $H$ captures the action of the full matrix $A$ on the Krylov space if does not venture too far outside the Krylov space, i.e. if there is some choice of coefficients $\left\{c_{j}\right\}$ for which $\left\|A v_{K-1}-\sum_{j=0}^{K-1} c_{j} v_{j}\right\|$ is sufficiently small. In this case the $K$ eigenvalues of $H$ are candidates for approximate eigenvalues of $A$. To capture complex eigenvalues, $K$ must be chosen such as to include both members of the pair.

The eigenvalues in Fig. 8 were obtained using the exponential power method (16), i.e. timestepping the linearized equations. This method is reliable and easy but, because the approximation requires a small timestep $\Delta t$, the operator resembles the identity and so the iteration proceeds slowly. In contrast, iteration using (18) is extremely rapid, converging to the eigenvector corresponding to the eigenvalue 
closest to zero, possibly on the order of 10 actions of the inverse. Each inverse action requires the solution of (18), typically via GMRES, BiCGSTAB or IDR. A shift is easily included in (18) by subtracting $s I$ from the explicit term $\mathcal{N}_{U}$, which finds eigenvalues close to $s$. using different shifts via the $s$. Figure 10(a) shows eigenvalues computed for spherical Couette flow (flow between differentially rotating concentric spheres) using different shifts via the inverse power method (18); see [22]. Figure 10(b), like Fig. 7, shows the speed of convergence in terms of number of actions of $\mathcal{B}_{T}$, and establishes that it is unchanged when the spatial resolution is increased.

A complex shift can be used to target eigenvalues on or close to the imaginary axis, which are those which lead to Hopf bifurcations. However, it is not clear how to scan large portions of the imaginary axis, if the frequencies being sought are unknown.

(a)

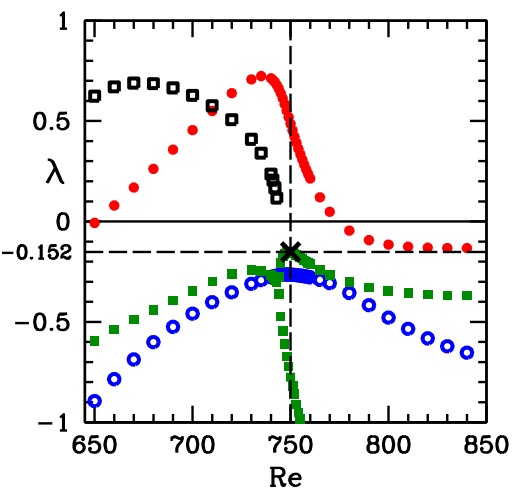

(b)

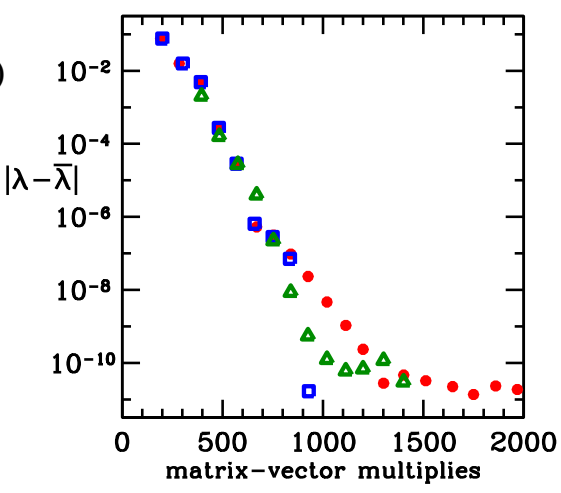

Fig. 10 (a) Four leading eigenvalues for spherical Couette flow. Two eigenvalues form a complex conjugate pair for $R e<744$; their real part is shown as solid green squares and the imaginary part as hollow black squares. The cross at $(R e=750, \lambda=-0.152)$ shows the targeted eigenvalue. (b) Convergence of error $|\lambda-\bar{\lambda}|$ as a function of Krylov space dimension and spatial resolution. Solid red circles: $K=2$ and spatial resolution $16 \times 128$. Hollow green triangles: $K=4$ and spatial resolution $16 \times 128$. Hollow blue squares: $K=2$ and $32 \times 256$. Reproduced from [22]

\section{Floquet analysis}

We now discuss the stability of periodic orbits $U(t)$, i.e. such that $U(t+T)=U(t)$. Infinitesimal perturbations $u$ to a periodic orbit $U(t)$ are governed by the equation

$$
\partial_{t} u=\mathcal{F}_{U(t)} u
$$

which is a Floquet problem, i.e. a linear differential equation whose coefficients are periodic in the independent variable, here time. Solutions to (19) are of the form 


$$
u(x, t)=\sum_{j} u_{j}(0) e^{\lambda_{j} t} \hat{v}_{j}(x, t \bmod T)
$$

where $x$ is used to designate any possible spatial dependence (which need not be present at all). $U(t)$ is stable if none of the Floquet exponents $\lambda_{j}$ have positive real part. Otherwise, $U(t)$ is unstable to perturbations of the form of the Floquet functions $\hat{v}_{j}(x, t \bmod T)$ corresponding to those $\lambda_{j}$ 's.

Floquet problems cannot generally be solved analytically, even when $U$ is a scalar and (19) is an ordinary differential equation. Floquet problems can be solved numerically in a variety of ways. One way is to integrate (19) starting from an initial condition $u(t=0)$ which is a unit vector, simultaneously with the determination of the limit cycle $U(t)$ by integrating (1). Assembling all the resulting vectors $u(t=T)$ leads to the monodromy matrix, whose eigenvalues are the Floquet multipliers $e^{\lambda_{j} T}$.

As is the case for ordinary stability problems, for problems with two or three spatial dimensions, it is usually too onerous to assemble and diagonalize the full monodromy matrix. The usual procedure is then to use Krylov methods, which compute a subset of the leading eigenvalues via a number $K$ of matrix actions where $K$ is much smaller than the total size of the system. The matrix action in this case is the integration of (19) over a time $T$.

We now present a sample of some large-scale Floquet problems which have been solved computationally. When a fluid layer is subjected to vertical oscillations of a sufficient amplitude, a standing-wave pattern forms on the surface, as was first studied by Faraday in 1831. The flat surface is a solution; the imposed vertical oscillation means that its destabilization is described by a Floquet problem. In an infinite horizontally homogeneous fluid layer, a solution is the product of a plane wave and a Floquet function

$$
\zeta(\mathbf{x}, t)=e^{i \mathbf{k} \cdot \mathbf{x}} \zeta(t)=e^{i \mathbf{k} \cdot \mathbf{x}} e^{\lambda t} \sum_{n} \zeta_{n} e^{i n \omega t}
$$

where $\zeta$ is the interface height. This Floquet problem has been solved by an original method [23]: rather than fixing the oscillation amplitude $a$ and calculating the resulting Floquet multipliers, the Floquet multiplier is set to $1=e^{0}$ (the harmonic case) or to $-1=e^{i \pi}$ (the subharmonic case), with the real part set to zero, thus determining the threshold $a_{c}$ directly. The method can be illustrated for the case of plane waves with fixed horizontal wavenumber $k$ on an inviscid fluid layer, for which the Floquet problem reduces to the Mathieu equation [24]:

$$
\ddot{\zeta}+\left(g k+\frac{\sigma}{\rho} k^{3}\right) \zeta=a \cos (\omega t) \zeta
$$

where $g$ is the gravitational acceleration, $\sigma$ is the surface tension, and $\rho$ is the density. In terms of the Fourier coefficients $\zeta_{n},(22)$ becomes

$$
\left((\lambda+i n \omega)^{2}+\left(g k+\frac{\sigma}{\rho} k^{3}\right)\right) \zeta_{n}=a[\cos (\omega t) \zeta]_{n}
$$


where $[\cdot]_{n}$ means the $n$th component of the expression within. Equation (23) is an eigenvalue problem with the set $\left\{\zeta_{n}\right\}$ as the eigenvector, $a$ as the eigenvalue, a diagonal matrix on the left-hand-side, and a bi-diagonal matrix on the right-handside. The inclusion of viscosity complicates the diagonal matrix on the left-hand-side but the basic idea of the method remains the same. Results obtained using this method are shown in Figure 11. These are compared with the threshold calculated from a full three-dimensional free-surface code.

(a)

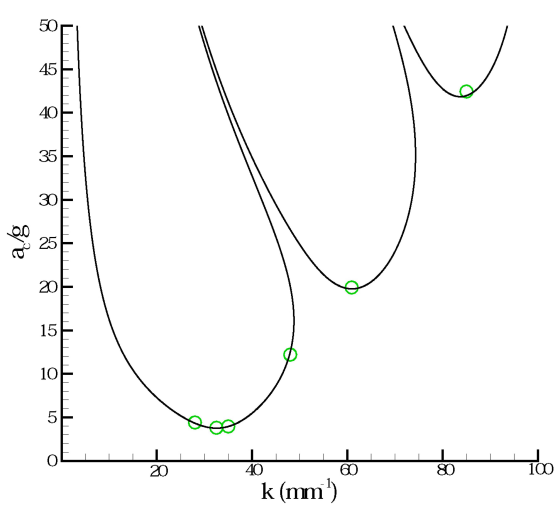

(b)
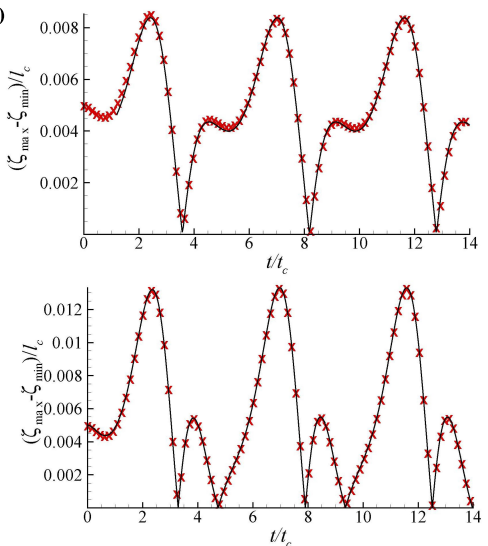

Fig. 11 (a) Critical acceleration for the onset of Faraday waves as a function of the wavenumber. The solid curves represent the neutral curves obtained by Floquet analysis, while the values found via full nonlinear simulation are indicated by the circles. (b) Linear evolution of the surface height of Faraday waves, in the first two instability tongues. Floquet functions are plotted with the solid line, while results from full nonlinear simulation near threshold are plotted with symbols. Reproduced from [25]

(a)

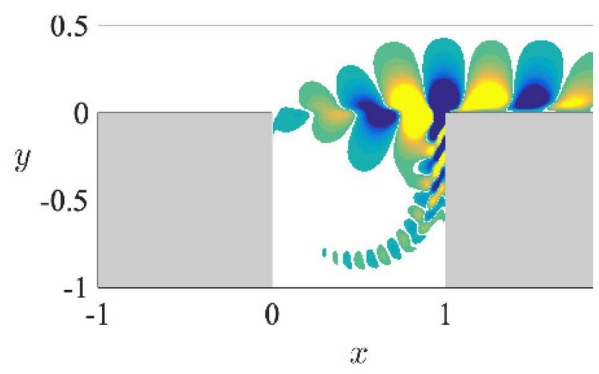

(b)

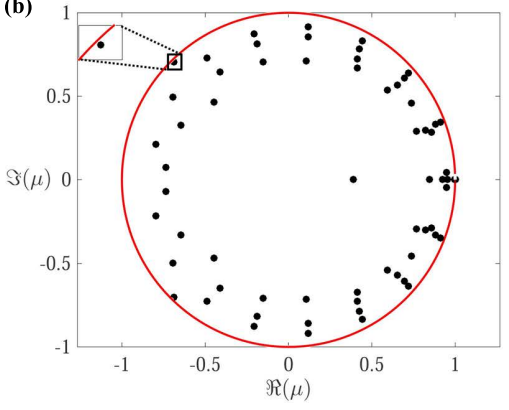

Fig. 12 (a) Instantaneous visualization of a temporally periodic state in an open shear-driven cavity at $R e=4500$. The vertical velocity fluctuations travel rightwards over the cavity. (b) Floquet multipliers of the periodic state in panel (a), emphasizing multiplier which traverses the unit circle at a slightly higher Reynolds number, thus destabilizing this periodic state. Reproduced from [26] 
One of the most classic hydrodynamic configurations is the wake of a circular cylinder in a uniform flow. Above a critical Reynolds number, a temporally periodic two-dimensional flow develops, in which vortices of alternating sign are generated close to the cylinder and advected downstream, a flow called the Bénard-von Kármán vortex street. Above a second critical Reynolds number, this spanwise-independent temporally periodic flow $\mathbf{U}_{2 D}(x, y, t$ mod $T$ ) becomes unstable to a spanwise periodic mode. This instability is governed by a Floquet problem, whose leading mode can be written as

$$
\mathbf{u}_{3 D}(x, y, z, t) \sim e^{i \beta z} e^{\lambda t} \mathbf{v}(x, y, t \bmod T)
$$

Barkley and Henderson [27] showed that the Bénard-von Kárman vortex street becomes unstable at $R e=188$ to a Floquet mode with spanwise wavelength of about four cylinder diameters and a Floquet exponent $\lambda=0$, meaning that no new time dependence is introduced into the resulting spanwise-dependent flow.

Another classic configuration is that generated by a uniform flow over a square cavity. Above a critical Reynolds number, a new flow appears which is temporally periodic and approximately spatially periodic in the streamwise direction, shown in Fig. 12a. A Floquet analysis [26] shows that above a second critical Reynolds number, this flow is in turn destabilized via a Floquet mode with a complex Floquet exponent, as shown in Fig. 12b.

\section{Heteroclinic orbits}

Among the more exotic phenomena displayed by dynamical systems are heteroclinic orbits: limit cycles whose period is infinite. Although the theory and understanding of heteroclinic orbits is well-grounded, they cannot be seen experimentally, since they are broken by any perturbation, and in fact can barely be seen computationally. Heteroclinic orbits can be divided into two broad categories. In one case, they occur at a single parameter value, at one end of the parameter range of existence of a limit cycle. Such a heteroclinic cycle is the signature of a global bifurcation which creates or destroys a limit cycle which, at neighboring parameter values, has a long but finite period. These cycles evade experimental observation because they occur only at isolated parameter values. Their existence is inferred from the divergence of the nearby periodic orbits. The other case is that of heteroclinic cycles which are robust, meaning that they exist over a range of parameters. These heteroclinic cycles result from symmetry properties. They evade experimental observation because symmetries in nature are rarely exact. 


\subsection{SNIPER bifurcations}

One mechanism by which heteroclinic cycles appear is via a SNIPER (SaddleNode In a PERiodic orbit, or Saddle-Node-Infinite-PERiod bifurcation) bifurcation $[28,29]$. On one side of the bifurcation, there exists a closed curve consisting of a chain of alternating stable and unstable fixed points connected by trajectories of the system. (In the simplest case, there is a single pair of stable and unstable fixed points; in a symmetric system, additional pairs exist.) The stable and unstable points meet and annihilate one another in saddle-node bifurcations, leaving in their wake a closed curve which forms one continuous closed trajectory, i.e. a limit cycle. At the bifurcation point $\mu_{c}$, the period of the limit cycle is infinite; slightly beyond $\mu_{c}$, the period behaves like $\left(\mu-\mu_{c}\right)^{-1 / 2}$.

A heteroclinic cycle was first observed for a full hydrodynamic configuration in a simulation of Rayleigh-Bénard convection in a cylinder [30]. In this case, the configuration is axisymmetric and the radius is five times the height. Two successive pitchfork bifurcations lead to four steady states, each consisting of a set of four or five concentric convection rolls, as shown in Fig. 13a. When the Rayleigh number is increased past the bifurcation point, the rolls begin to travel radially inwards, as shown in Fig. 13c: the innermost roll shrinks and disappears, while a new roll appears at the outer boundary.

SNIPER bifurcations have been computed in other systems. In another axisymmetric cylindrical convective configuration, the top and bottom disks bounding the domain are rotated at equal and opposite speeds (the von Kármán flow described below in section 5.2), in addition to having fixed and different temperatures [31]. The height and radius are equal, with one large concentric roll filling almost the entire domain. During the cycle a small roll appears alternatively at the outer radial boundary, alternating between the top and the bottom of the cylinder. The limit cycle exists in a region of the (Reynolds number, Rayleigh number) parameter plane delimited by a SNIPER bifurcation curve and a Hopf bifurcation curve. SNIPER bifurcations have also been observed in simulations of other variants of cylindrical Rayleigh-Bénard convection [32, 33].

\subsection{1:2 Mode Interaction}

Turning now to robust heteroclinic cycles, i.e. those which exist over a range of parameters, one important mechanism is provided by the 1:2 mode interaction. This scenario occurs in a configuration with $O(2)$ symmetry, when pitchfork bifurcations to modes with azimuthal wavenumbers $m=1$ and $m=2$ modes occur at close parameter values. In this case, Armbruster et al. [34] predict the existence of $m=1$ and $m=2$ steady states, traveling waves, modulated traveling waves, and robust heteroclinic cycles. These cycles are trajectories between two $m=2$ states that differ by a change in phase of $\pi / 2$. Several full hydrodynamic computations have displayed realizations of this 1:2 mode interaction scenario. Nore et al. [13] investigated the 

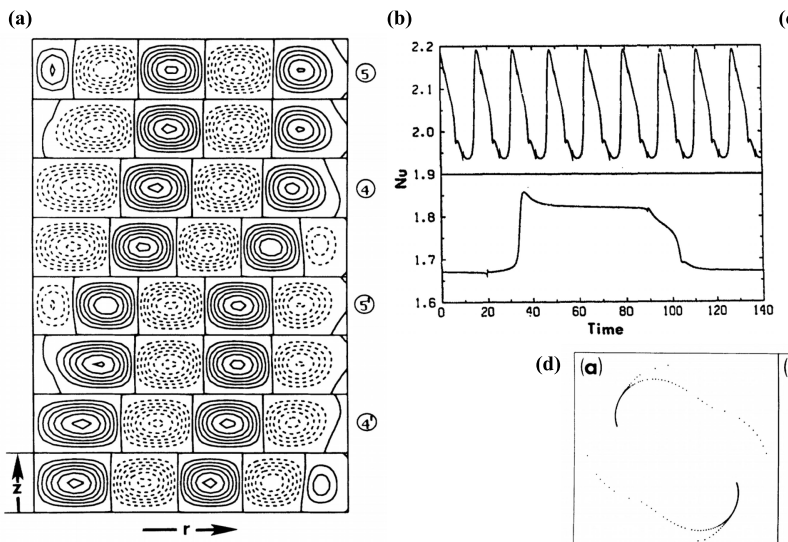

(c)

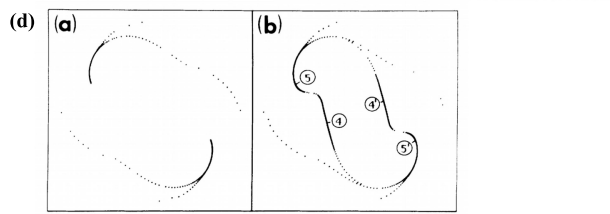

Fig. 13 Cylindrical convection. (a) Instantaneous streamfunction during the limit cycle at $\epsilon=1.39$ at successive times. The numbers label slowly varying quasi five-roll and quasi four-roll states. (b) Nusselt number time series for $\epsilon=2.60$ (above) and for $\epsilon=1.39$ (below). The period diverges at the SNIPER bifurcation. (c) Schematic bifurcation diagram using the reduced Rayleigh number $\epsilon$ as the control parameter and a projection $A$ of the states as order parameter. Above (a, b) are numerically calculated streamfunction contours of representative five-roll steady states. Below (c-g) are phase portraits at the values of $\epsilon$ denoted by tick marks. Solid lines and filled circles denote stable states, while dashed lines and hollow circles denote unstable states. The straight bold curve represents the limit cycle formed in the SNIPER bifurcation. (d) Phase portraits using as coordinates the projections onto the two most unstable eigenvectors of the conductive state. (a) is computed at $\epsilon=1.38$ [corresponding to (f) in panel (c)] and at (b) $\epsilon=1.39$ [corresponding to (g) in panel (c)]. All trajectories in (a) terminate at one of two steady states. Numbers in (b) correspond to visualizations of limit cycle in panel (c). Reproduced from [30]

flow in a cylinder whose height is twice the radius and whose two bounding disks cylinder rotate in equal and opposite directions, sometimes called von Kármàn flow. The basic state is axisymmetric and consists of an axial shear of the azimuthal velocity combined with two superposed toroidal cells driven by Ekman pumping and separated at the midplane between the top and bottom disks. The flow undergoes circle pitchfork bifurcations which break the axisymmetry, leading to an undulation in the separating surface and to radially oriented vortices.

Figure 14(b) shows results of linear stability analysis, in particular, the $m=1$ and $m=2$ modes (called mixed $\mathrm{M}$ and pure $\mathrm{P}$, respectively, because the higher harmonics of $m=1$ contain both odd and even wavenumbers, while those of $m=2$ contain only even wavenumbers). The modes are represented visually via the vertical velocity at the midplane. Figure 14(b) shows the sequence of bifurcations in this case, adapted from those predicted in [34]. The mixed branch engenders traveling waves (via a drift pitchfork bifurcation) and then modulated traveling waves (via a Hopf bifurcation). The branch of pure states is unstable at onset, but engenders a heteroclinic cycle that consists of alternation between states which are $2 \pi / 4$ out of azimuthal phase from one another. 
Returning to Fig. 14(a), many of these transitions coincide with the crossings of eigenvalues, as explained in the AGH scenario. The left part shows the result of linearization about the basic state; the mixed and pure branches are created via pitchfork bifurcations when the $m=1$ and $m=2$ eigenvalues cross zero. The right part shows the result of linearization about the pure states, when these exist. Phase becomes significant and so the eigenmodes of the basic state each split into those symmetric (S) and antisymmetric (A) with respect to the pure mode. The $m=2$ eigenmodes split into the classic negative amplitude and neutral phase modes. The $m=1$ eigenvalues decrease; the mixed branch is annihilated when the eigenvalue associated with $1 \mathrm{~S}$ becomes negative and when its magnitude surpasses that of the still-positive eigenvalue associated with $1 \mathrm{~A}$, the heteroclinic cycle becomes stable. Excursions consist of growth along the 1A direction and decay along the $1 \mathrm{~S}$ direction. When the eigenvalue associated with $1 \mathrm{~A}$ becomes negative, the pure mode becomes stable. This case shows the power of combining full nonlinear timedependent simulations with computational linear stability analysis.
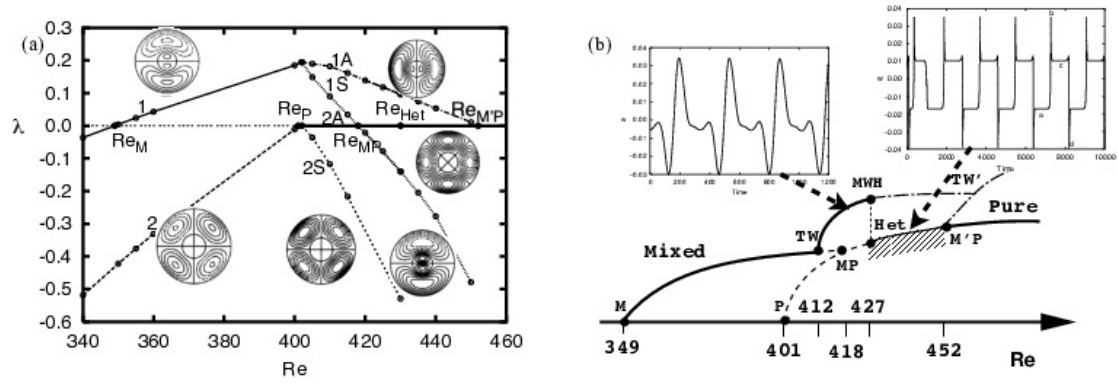

Fig. 14 The 1:2 mode interaction in counter-rotating von Kármán flow. (a) Eigenvalues corresponding to azimuthal wavenumbers $m=1$ and 2 of the basic state for $R e<R e_{P}$, the threshold of bifurcation to the pure mode state, and of the pure mode state for $R e>R e_{P}$. Contours of vertical velocity of each eigenvector are shown. (b) Schematic bifurcation diagram showing bifurcations: circle pitchforks from the basic branch to mixed $(\mathrm{M})$ and pure $(\mathrm{P})$ branches, drift to traveling waves (TW), Hopf to modulated waves (MWH), pitchforks (MP, M'P) connecting mixed and pure branches. The heteroclinic orbit is stable between thresholds Het and M'P. Timeseries of traveling waves and the heteroclinic cycle are shown. Adapted from [13]

Heteroclinic cycles associated with the 1:2 mode interaction have been computed in other hydrodynamic systems. Mercader et al. [36] simulated non-Boussinesq two-dimensional Rayleigh-Bénard convection in a rectangle of width $\pi$ (between the favored wavelengths of approximately 2 and 4). Bengana and Tuckerman [35] simulated Taylor-Couette flow between counter-rotating cylinders and discovered two very different cycles, one whose excursions resemble the non-axisymmetric azimuthally traveling ribbon state, and another whose excursions remain axisymmetric, shown via the phase portraits in Fig. 15. Both cycles are based at the same saddles and the crossover between them corresponds to the crossover between two leading 
a)

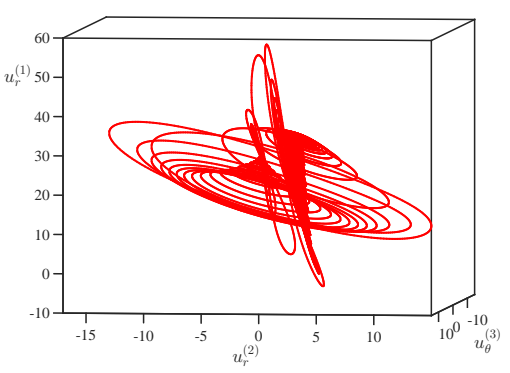

b)

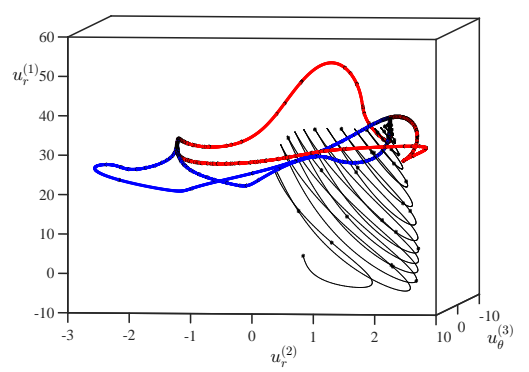

Fig. 15 Phase portraits of two heteroclinic orbits in counter-rotating Taylor-Couette flow. a) Excursions are non-axisymmetric and spiral in and out of the saddles. b) After a spiralling initial transient, the excursions are non-oscillatory and chosen quasi-randomly from several possible trajectories. Both cycles are anchored by the same saddles. Reproduced from [35]

eigenvalues, that corresponding to $1 \mathrm{~A}$ and a complex eigenvalue corresponding to a non-axisymmetric eigenmode. In all of these computations, the cycles may behave in a regular or slightly irregular way. Their period is not infinite, so strictly speaking, they should be called near-heteroclinic cycles.

All of the cycles described above described were calculated using only timeintegration. However, there exist more specialized numerical methods aimed specifically at calculating heteroclinic cycles [37, 38, 39]

\section{Hamiltonian Systems: Bose-Einstein Condensation}

A Bose-Einstein condensate can be represented by a complex wave function $\Psi(\mathbf{x}, t)$ that obeys the Gross-Pitaevskii equation

$$
-i \partial_{t} \Psi=\left[\frac{1}{2} \nabla^{2}-V(x)-a|\Psi|^{2}+\mu\right] \Psi
$$

where $a$ is the nondimensionalized scattering length and and

$$
V(x) \equiv \frac{1}{2}|\omega \cdot \mathbf{x}|^{2}
$$

is a confining harmonic potential. The Lagrange multiplier $\mu$ is associated with the constraint that the particle number $\mathcal{N} \equiv \int|\Psi|^{2} d^{3} x$ be kept constant. (In this section, we use $\mathcal{N}$ to denote particle number, in contrast to sections 1-3, in which it denotes the nonlinear terms).

We have considered a potential with cylindrical symmetry, i.e. with inverse length scales $\omega_{x}=\omega_{y}=\omega_{r}$ and $\omega_{z}$, in either the pancake $\left(\omega_{r}<\omega_{z}\right)$ or cigar $\left(\omega_{r}>\omega_{z}\right)$ configuration. Equation (25) is distinguished from the equations considered in the previous sections by the presence of $i$. As a Hamiltonian system, its bifurcations are different from those of dissipative systems. With the cylindrical potential, we 


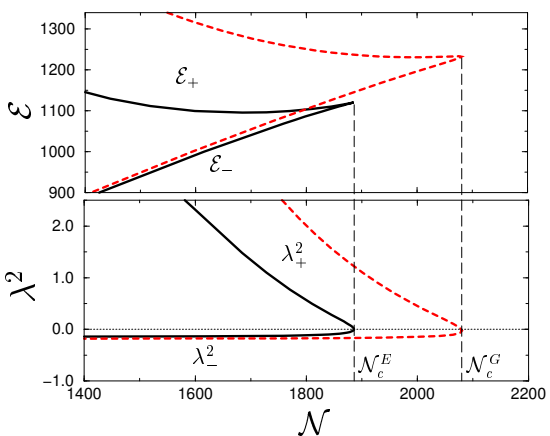

Fig. 16 Stationary solutions of the GP equation as a function of the particle number $\mathcal{N}$ for a potential (26) of pancake form with $\omega_{z}=5 \omega_{r}$. Energy functional (above) and square of the bifurcating eigenvalue $\lambda_{+}^{2}$ (below). Exact numerical solution (black solid lines) is compared with Gaussian approximation (red dashed lines). Reproduced from [40]

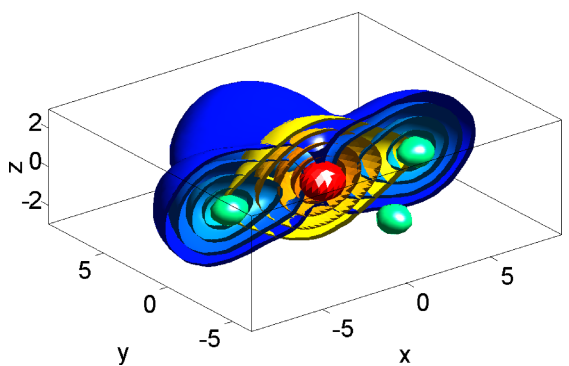

Fig. 17 Stable symbiotic vortex-bright structure in the presence of an optical lattice with potential (34) with $\omega_{z}=5 \omega_{r}$. Surfaces of constant density are shown in blue for the vortex and in yellow for the soliton. This 3D stationary state is stabilized by the second component, which displaces the vortex component at its core. Reproduced from [41]

find that (25) exhibits a Hamiltonian saddle-node bifurcation. A saddle and a center coexist and annihilate one another at a critical value of $\mu$ or, equivalently, $\mathcal{N}$.

The search for steady states is not affected by the presence of $i$; we merely solve

$$
0=\left[\frac{1}{2} \nabla^{2}-V(\mathbf{x})-a|\Psi|^{2}+\mu\right] \Psi
$$

This is done via the same technique as in section 5, with $\mathcal{L} \Psi=\frac{1}{2} \nabla^{2} \Psi$ and $\mathcal{N}=$ $-\left(V(x)+a|\Psi|^{2}-\mu\right) \Psi$. These steady states are shown for the pancake case via their energy

$$
\mathcal{E} \equiv \int d^{3} x\left[\frac{1}{2}|\nabla \Psi|^{2}+V(\mathbf{x})|\Psi|^{2}+\frac{a}{2}|\Psi|^{4}\right]
$$

in the upper part of Fig. 16. We mention some other methods that have been used to compute such states. For a spherically symmetric potential $\omega_{r}=\omega_{z}$, the wavefunction is one-dimensional, so that linear systems arising in Newton's method are much smaller and can easily be solved directly. One method approximates the functional dependence of $\Psi(\mathbf{x})$ as Gaussian; this yields a problem without any spatial dependence in which the few scalar parameters of this approximation are computed via Newton's method. Figure 16 shows the resulting branches, which are qualitatively, but not quantitatively, the same as the exact solutions. Another method, called relaxation or imaginary time, integrates 


$$
\partial_{t} \Psi=\left[\frac{1}{2} \nabla^{2}-V(x)-a|\Psi|^{2}+\mu\right] \Psi
$$

turning the system into a dissipative one. However, this method does not find the branches which are unstable under the evolution of (29), and so cannot obtain the full diagram of Fig. 16.

We now turn to the stability of the real steady states $\Psi$ of (25). The eigenmodes $\left(\lambda, \psi_{R}, \psi_{I}\right)$ satisfy

$$
\lambda\left(\begin{array}{l}
\psi_{R} \\
\psi_{I}
\end{array}\right)=\left[\begin{array}{cc}
0 & -\left(\mathcal{L}+D W_{I}\right) \\
\mathcal{L}+D W_{R} & 0
\end{array}\right]\left(\begin{array}{l}
\psi_{R} \\
\psi_{I}
\end{array}\right)
$$

where

$$
\mathcal{L} \Psi \equiv \frac{1}{2} \nabla^{2} \Psi, \quad D W_{R} \equiv \mu-V(\mathbf{x})-3 a \Psi^{2}, \quad D W_{I} \equiv \mu-V(\mathbf{x})-a \Psi^{2} .
$$

The eigenvalues occur in equal and opposite pairs and the pair closest to zero determines the nature of each branch. For states on the stable elliptic branch $\mathcal{E}_{-}$, this pair is imaginary, so $\lambda^{2}$ is negative; $\left|\lambda_{-}\right|$is the energy of excitations around this branch. Along the unstable hyperbolic branch $\mathcal{E}_{+}$, this pair is real, so that $\lambda_{+}^{2}$ is positive. At the saddle-node bifurcation, these eigenvalues meet at zero. All of the other eigenvalues are imaginary pairs.

In order to calculate the critical eigenvalue pair, we have used the inverse power method (see section 3). Moreover, we square the matrix, which leads to a block diagonal matrix whose blocks both have the same eigenvalues $\lambda^{2}$ :

$$
\lambda^{2}\left(\begin{array}{l}
\psi_{R} \\
\psi_{I}
\end{array}\right)=\left[\begin{array}{cc}
-\left(\mathcal{L}+D W_{I}\right)\left(\mathcal{L}+D W_{R}\right) & 0 \\
0 & -\left(\mathcal{L}+D W_{R}\right)\left(\mathcal{L}+D W_{I}\right)
\end{array}\right]\left(\begin{array}{l}
\psi_{R} \\
\psi_{I}
\end{array}\right)
$$

Preconditioning by $\mathcal{L}^{-2}$, we carry out the inverse square iteration via:

$$
-\mathcal{L}^{-2}\left(\mathcal{L}+D W_{I}\right)\left(\mathcal{L}+D W_{R}\right) \psi_{R}^{(n+1)}=\mathcal{L}^{-2} \psi_{R}^{(n)}
$$

These methods and results are described in detail in [11, 40].

In a separate investigation, $\Psi$ is prolonged to include a second complex component and a periodic lattice component is added to the confining potential

$$
V(r, z)=\frac{1}{4}|\omega \cdot \mathbf{x}|^{2}+A\left[\sin ^{2}\left(2 \omega_{r} x\right)+\sin ^{2}\left(2 \omega_{r} y\right)\right]
$$

leading to a problem which is not axisymmetric. Using the steady-state methods described above, we have computed a non-axisymmetric solution consisting of stable symbiotic vortex-bright solitons shown in Fig. 17; see [41]. 


\section{Looking ahead}

In this chapter, we have described a number of dynamical-systems phenomena, namely steady states and limit cycles; bifurcations, instability and Floquet analysis; and heteroclinic cycles, that occur in many nonlinear physical systems. Many of these phenomena, such as unstable steady states or heteroclinic orbits, are accessible only to computation.

The numerical simulations described in the previous sections have uncovered several new dynamical-systems scenarios. Faraday waves provide an example for which full nonlinear three-dimensional simulations have led to the discovery of new phenomena. First, in simulations of Faraday waves in a minimal hexagonal domain [42], the hexagons give way after many subharmonic oscillation periods to a pattern we have called beaded stripes. These are succeeded in turn by quasi hexagons, and then by regular alternation between asymmetric beaded stripes and quasi hexagons, as shown in Fig. 18. The bifurcation-theoretic genesis of this complicated scenario is unknown. Secondly [43], a large square domain containing Faraday waves spontaneously divides into a two-by-two grid in which the square waves are in phase with those diagonally opposite, as shown in Fig. 19. Finally, Faraday waves on the surface of a drop induced by a radially directed oscillatory force display patterns [44], many of which resemble Platonic solids, as expected [45]. However, some of the patterns we observe undergo a slow and long-lasting drift in orientation, such as the axisymmetric pattern of Fig. 20. This feature remains unexplained. Although bifurcation analysis has been carried out for thin films [46], most tools for numerical bifurcation analysis have not yet been applied to full three-dimensional nonlinear free-surface problems.

In most quantitative sciences, a surprisingly important bottleneck is that of numerical linear algebra, and this is certainly the case for nonlinear dynamics. The main focus of computational science is time-integration, which has been tamed to yield algorithms whose timing is approximately linear in the size $M$ of the system. Over the years, many monographs, e.g. [48, 49, 50, 51], dedicated volumes, e.g. $[52,53,54]$, review articles, e.g. $[15,55,56]$, and software packages such as AUTO [57], DSTOOL [58], PDECont [59], MatCont [60], LOCA [61], JuliaDynamics [62], pde2path [63] have been dedicated to targeting simple or complicated bifurcation-theoretic objects for general dynamical systems. However, such algorithms usually require the solution of linear systems or matrix diagonalization. Straightforward algorithms for these tasks scale like $M^{3}$, and if the matrix is derived from a two or three-dimensional partial differential equation then $M=M_{x} M_{y} M_{z}$ may be on the order of $10^{6}$ or more. Krylov space methods can be used, in which the solution is obtained as a superposition of $K$ vectors obtained by matrix actions. The timings for these scale like $K M$ or $K^{2} M$, so the crucial challenge is to keep $K$ low.

A number of strategies for speeding up the iterative solution of linear systems have been proposed in this chapter, more specifically transforming the matrix or the nonlinear problem to one which is more tractable. These include using the exponential or the inverse of the operator and preconditioning by the Laplacian. An extension of this approach is as follows. Consider a steady-state problem containing 


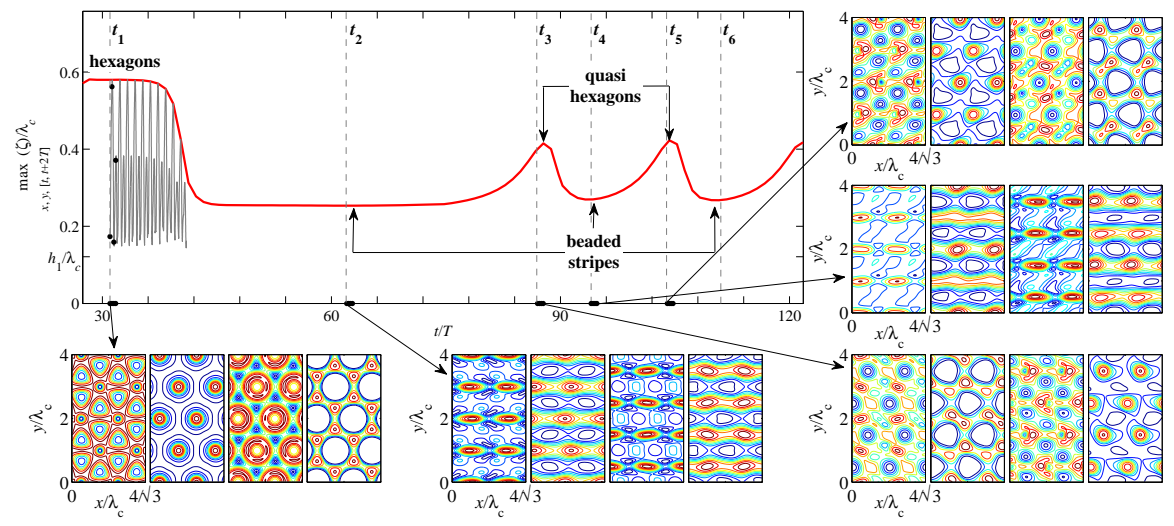

Fig. 18 Complex dynamics beginning with hexagons at time $t_{1}$, beaded stripes at time $t_{2}$, quasihexagons at $t_{3}$ and $t_{5}$, and asymmetric beaded stripes at $t_{4}$ and $t_{6}$. Curves show maxima of interface height, with the rapidly oscillating curve showing $\max _{x, y} \zeta(x, y, t)$ for initial times and the smooth curve showing $\max _{x, y,[t, t+T]} \zeta(x, y, t)$. Surrounding visualizations show instantaneous contour plots of $\zeta(x, y, t)$ at four temporal phases within one oscillation period. The size of the box has been doubled in each dimension. Over the large white areas, the interface is very close to the bottom and almost flat. Reproduced from [47]. Creative Commons Attribution License (CC BY) https://creativecommons.org/licenses/by/4.0/

a)

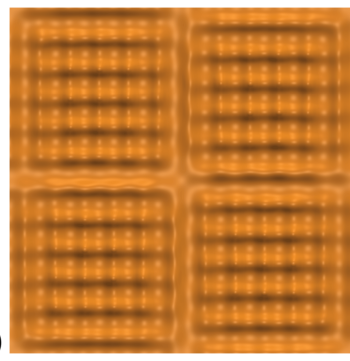

b)

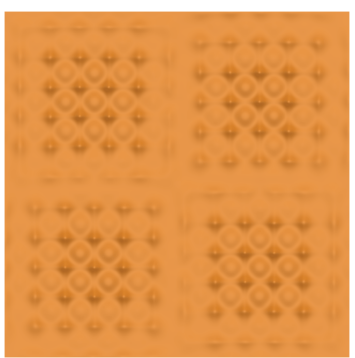

c)

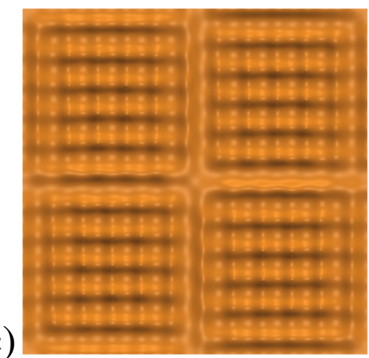

Fig. 19 Supersquare pattern at instants separated by intervals of $T / 2$, where $T$ is the forcing period and $2 T$ is the subharmonic response period. In (a) and (c), wells are surrounded by ridges with peaks at each corner, while in (b), the squares are centered around low peaks. The large squares on the bottom left and top right are in phase, as are those on the bottom right and top left. Reproduced from [43]

a linear operator $\mathcal{L}$ and a bilinear operator $\mathcal{N}$.

$$
0=\mathcal{F}(U) \equiv \mathcal{L} U+\mathcal{N}(U, U)
$$

In order to compute $u$, an improvement to the estimate $U$, Newton's method requires us to solve

$$
\begin{aligned}
\mathcal{F}_{U} u & =F(U) \\
\mathcal{L} u+\mathcal{N}(U, u)+\mathcal{N}(u, U) & =\mathcal{L} U+\mathcal{N}(U, U)
\end{aligned}
$$




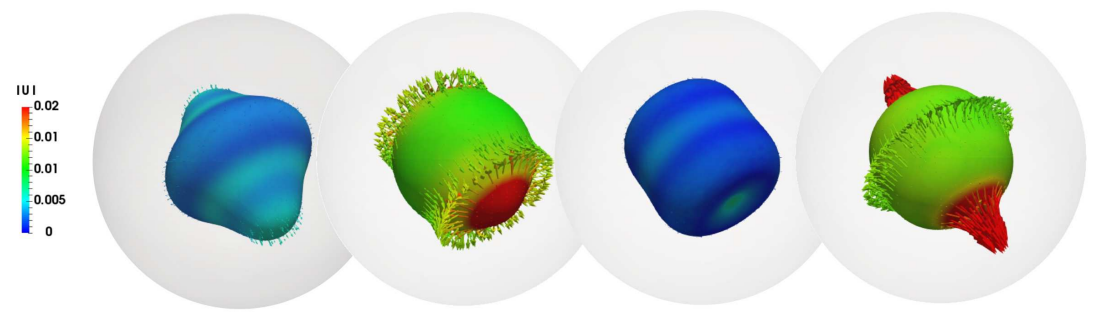

Fig. 20 Axisymmetric $\ell=4$ pattern seen in capillary waves during one subharmonic response period $2 T$. The interface sometimes resembles a top and sometimes a finite cylinder. Each fluid drop is surrounded by its spherical domain. The magnitude of the velocity is indicated by the colors. Reproduced from [44]

where $u$ is a decrement to $U$ that should decrease $\|\mathcal{L} U+\mathcal{N}(U)\|$. The Stokes method described in section 2 preconditions (36) with $\mathcal{L}^{-1}$

$$
\mathcal{L}^{-1}[\mathcal{L} u+\mathcal{N}(U, u)+\mathcal{N}(u, U)]=\mathcal{L}^{-1}[\mathcal{L} U+\mathcal{N}(U, U)]
$$

It is easy to generate other decompositions of (35) that lead to other preconditioners. For any fixed field $U_{\text {base }}$, we may write

$$
U=U_{\text {base }}+\tilde{U}
$$

The operator whose roots are sought is unchanged, but it now formally acts on the unknown $\tilde{U}$

$$
F(\tilde{U})=\mathcal{L} U_{\text {base }}+\mathcal{L} \tilde{U}+\mathcal{N}\left(U_{\text {base }}, U_{\text {base }}\right)+\mathcal{N}\left(U_{\text {base }}, \tilde{U}\right)+\mathcal{N}\left(\tilde{U}, U_{\text {base }}\right)+\mathcal{N}(\tilde{U}, \tilde{U})
$$

and has Jacobian

$$
D F_{\tilde{U}} \tilde{u}=\mathcal{L} \tilde{u}+\mathcal{N}\left(U_{\text {base }}, \tilde{u}\right)+\mathcal{N}\left(\tilde{u}, U_{\text {base }}\right)+\mathcal{N}(\tilde{U}, \tilde{u})+\mathcal{N}(\tilde{u}, \tilde{U})
$$

where $\tilde{u}$ is a decrement to $\tilde{U}$ that should decrease $F(\tilde{U})$. If $U_{\text {base }}$ is sufficiently simple, it may be possible to invert

$$
\mathcal{L}+\mathcal{N}\left(U_{\text {base }}, \cdot\right)+\mathcal{N}\left(\cdot, U_{\text {base }}\right)
$$

The inverse of (41) may be a much better preconditioner for $F$ than $\mathcal{L}^{-1}$ since it includes parts of $\mathcal{N}$ which were previously left out of the preconditioning. As an example of this approach, [18] discusses the computation of steady states and traveling waves in wall-bounded shear flows. For such flows, there is a known laminar base flow $\mathbf{U}_{\text {base }}=U(y) \mathbf{e}_{x}$ for which

$$
\mathbf{U} \cdot \nabla \mathbf{u}+\mathbf{u} \cdot \nabla \mathbf{U}=U(y) \partial_{x} \mathbf{u}+u_{y} \partial_{y} U(y) \mathbf{e}_{x}
$$


Incorporating one or both of these terms into the Stokes preconditioner would almost surely greatly lower the number $K$ of Krylov vectors needed.

Another way in which the search for steady states may be modified is by applying an operator or functional $\mathcal{G}$ to $\mathcal{F}$, transforming the steady-state problem to

$$
0=\mathcal{G}(0)-\mathcal{G}(\mathcal{F}(U))
$$

Again, this is advantageous if the Jacobian of $\mathcal{G}(\mathcal{F})$, i.e. $\mathcal{G F}_{\mathcal{F}} \mathcal{F}_{U}$ is better conditioned than $\mathcal{F}_{U}$, or if a better preconditioner for it is available. In fact the two approaches (11) and (12) constitute a special case of such a transformation, where $\mathcal{G}$ is taken to be the integral of $\mathcal{F}$

$$
\mathcal{G}(\mathcal{F}(U)) \equiv \int_{t}^{t+T} \mathcal{F}(U(\tau)) d \tau
$$

Similarly, if $\mathcal{F}$ is decomposed into $\mathcal{L}+\mathcal{N}$, as in (35), then applying $\mathcal{G}$ leads to the problem

$$
0=\mathcal{G}(\mathcal{L}(U))-\mathcal{G}(-\mathcal{N}(U))
$$

which could be preconditioned by $[\mathcal{G}(\mathcal{L})]^{-1}$ if $\mathcal{G}$ is linear. It would be desirable to popularize such strategies and to augment these by finding new ones, or, even better, to automate the search for an optimal transformation.

\section{References}

1. B. Hof, P.G. Lucas, T. Mullin, Phys. Fluids 11, 2815 (1999)

2. K. Borońska, L.S. Tuckerman, Phys. Rev. E 81, 036320 (2010)

3. K. Borońska, L.S. Tuckerman, Phys. Rev. E 81, 036321 (2010)

4. Y. Saad, M.H. Schultz, SIAM J. Sci. Stat. Comput. 7, 856 (1986)

5. H.A. Van der Vorst, SIAM J. Sci. Stat. Comput. 13, 631 (1992)

6. P. Sonneveld, M.B. Van Gijzen, SIAM J. Sci. Comput. 31, 1035 (2008)

7. L.S. Tuckerman, in 11th International Conference on Numerical Methods in Fluid Dynamics, ed. by D.L. Dwoyer, M.Y. Hussaini, R.G. Voight. Lecture Notes in Physics, Vol. 323 (Springer, Berlin Heidelberg, 1989), p. 573

8. C.K. Mamun, L.S. Tuckerman, Phys. Fluids 7, 80 (1995)

9. S. Xin, P. Le Quéré, L.S. Tuckerman, Phys. Fluids 10, 850 (1998)

10. A. Bergeon, D. Henry, H. Benhadid, L.S. Tuckerman, J. Fluid Mech. 375, 143 (1998)

11. C. Huepe, S. Metens, G. Dewel, P. Borckmans, M.E. Brachet, Phys. Rev. Lett. 82, 1616 (1999)

12. L.S. Tuckerman, D. Barkley, in Numerical methods for bifurcation problems and large-scale dynamical systems, ed. by E. Doedel, L.S. Tuckerman. The IMA Volumes in Mathematics and its Applications, Vol. 119 (Springer, New York, 2000), p. 453

13. C. Nore, L.S. Tuckerman, O. Daube, S. Xin, J. Fluid Mech. 477, 51 (2003)

14. I. Mercader, A. Alonso, O. Batiste, Eur. Phys. J. E 15, 311 (2004)

15. H.A. Dijkstra, F.W. Wubs, A.K. Cliffe, E. Doedel, I.F. Dragomirescu,B. Eckhardt, A.Y. Gelfgat, A.L. Hazel, V. Lucarini, A.G. Salinger, E.T. Phipps, J. Sanchez-Umbria, H. Schuttelaars, L.S. Tuckerman, U. Thiele, Commun. Comput. Phys. 15, 1 (2014)

16. P. Cvitanović, B. Eckhardt, J. Phys. A 24, L237 (1991)

17. J.F. Gibson, J. Halcrow, P. Cvitanović, J. Fluid Mech. 638, 243 (2009) 
18. L.S. Tuckerman, J. Langham, A. Willis, in Computational Modelling of Bifurcations and Instabilities in Fluid Dynamics, ed. by A. Gelfgat (Springer, Cham, 2019), p. 3

19. J.F. Gibson, Channelflow: A spectral Navier-Stokes simulator in C++ (2014), http: //channelflow.org. Accessed 18 December 2019

20. A.P. Willis, SoftwareX 6, 124 (2017)

21. C.C. Pringle, Y. Duguet, R.R. Kerswell, Phil. Trans. Royal Soc. A 367, 457 (2009)

22. L.S. Tuckerman, Commun. Comput. Phys. 18, 1336 (2015)

23. K. Kumar, L.S. Tuckerman, J. Fluid Mech. 279, 49 (1994)

24. T.B. Benjamin, F.J. Ursell, Proc. R. Soc. A 225, 505 (1954)

25. N. Perinet, D. Juric, L.S. Tuckerman, J. Fluid Mech. 635, 1 (2009)

26. Y. Bengana, J.C. Loiseau, J.C. Robinet, L.S. Tuckerman, J. Fluid Mech. 875, 725 (2019)

27. D. Barkley, R.D. Henderson, J. Fluid Mech. 322, 215 (1996)

28. A. Andronov, E. Leontovich, Matem. Sb. 48, 335 (1959)

29. Y.A. Kuznetsov, Elements of Applied Bifurcation Theory (Springer, New York, 2004)

30. L.S. Tuckerman, D. Barkley, Phys. Rev. Lett. 61, 408 (1988)

31. L. Bordja, L.S. Tuckerman, L.M. Witkowski, M.C. Navarro, D. Barkley, R. Bessaih, Phys. Rev. E 81, 036322 (2010)

32. J.M. Lopez, A. Rubio, F. Marques, J. Fluid Mech. 569, 331 (2006)

33. J.H. Siggers, J. Fluid Mech. 475, 357 (2003)

34. D. Armbruster, J. Guckenheimer, P. Holmes, Physica D 29, 257 (1988)

35. Y. Bengana, L.S. Tuckerman, Phys. Rev. Fluids 4, 044402 (2019)

36. I. Mercader, J. Prat, E. Knobloch, Int. J. Bifurcat. Chaos 12, 2501 (2002)

37. J. Halcrow, J.F. Gibson, P. Cvitanović, D. Viswanath, J. Fluid Mech. 621, 365 (2009)

38. L. Van Veen, G. Kawahara, M. Atsushi, SIAM J. Sci. Comput. 33, 25 (2011)

39. M. Farano, S. Cherubini, J.C. Robinet, P. De Palma, T.M. Schneider, J. Fluid Mech. 858 (2019)

40. C. Huepe, L.S. Tuckerman, S. Métens, M.E. Brachet, Phys. Rev. A 68, 023609 (2003)

41. K.J. Law, P.G. Kevrekidis, L.S. Tuckerman, Phys. Rev. Lett. 105, 160405 (2010)

42. N. Périnet, D. Juric, L.S. Tuckerman, Phys. Rev. Lett. 109, 164501 (2012)

43. L. Kahouadji, N. Périnet, L.S. Tuckerman, S. Shin, J. Chergui, D. Juric, J. Fluid Mech. 772 (2015)

44. A.H. Ebo-Adou, L.S. Tuckerman, S. Shin, J. Chergui, D. Juric, J. Fluid Mech. 870, 433 (2019)

45. P. Chossat, R. Lauterbach, I. Melbourne, Arch. Ration. Mech. An. 113, 313 (1991)

46. P. Beltrame, U. Thiele, SIAM J. Appl. Dyn. Syst. 9, 484 (2010)

47. N. Périnet, D. Juric, L.S. Tuckerman, Rev. Cuba. Fís. 29, 1E6 (2012)

48. M. Kubicek, M. Marek, Computational methods in bifurcation theory and dissipative structures (Springer, Berlin Heidelberg, 1983)

49. H.B. Keller, Lectures on Numerical Methods in Bifurcation Problems. Lectures on Mathematics and Physics Mathematics, vol. 79 (Springer, Berlin Heidelberg, 1988)

50. R. Seydel, From Equilibrium to Chaos: Practical Bifurcation and Stability Analysis (Elsevier, Amsterdam, 1988)

51. W.J.F. Govaerts, Numerical Methods for Bifurcations of Dynamical Equilibria (SIAM, Philadelphia, 2000)

52. E. Doedel, L.S. Tuckerman (eds.), Numerical Methods for Bifurcation Problems and LargeScale Dynamical Systems. The IMA Volumes in Mathematics and its Applications, Vol. 119 (Springer, New York, 2000)

53. B. Krauskopf, H.M. Osinga, J. Galán-Vioque (eds.), Numerical Continuation Methods for Dynamical Systems (Springer Netherlands, 2007)

54. A.S. Dirk Roose, Bart De Dier, A. Spence (eds.), Continuation and Bifurcations: Numerical Techniques and Applications. NATO Science Series C, Vol. 313 (Springer Netherlands, 1990)

55. K.A. Cliffe, A. Spence, S.J. Tavener, Acta Numer. 9, 39 (2000)

56. E.J. Doedel, in Numerical Continuation Methods for Dynamical Systems, ed. by B. Krauskopf, H.M. Osinga, J. Galán-Vioque (Springer Netherlands, 2007), p. 1

57. E.J. Doedel, Congr. Numer. 30, 265 (1981)

58. A. Back, J. Guckenheimer, M. Myers, F. Wicklin, P. Worfolk, Not. Am. Math. Soc. 39, 303 (1992) 
59. K. Lust, D. Roose, SIAM J. Sci. Comput. 19, 1188 (1998)

60. A. Dhooge, W. Govaerts, Y.A. Kuznetsov, ACM Trans. Math. Software 29, 141 (2003)

61. A.G. Salinger, N.M. Bou-Rabee, R.P. Pawlowski, E.D. Wilkes, E.A. Burroughs, R.B. Lehoucq, L.A. Romero, LOCA 1.0 Library of continuation algorithms: theory and implementation manual. Tech. Rep. SAND2002-0396, Sandia National Laboratories (2002)

62. G. Datseris, J. Open Source Softw. 3, 598 (2018)

63. H. Uecker, D. Wetzel, J.D.M. Rademacher, Numer. Math. Theory Me. 7, 58 (2014) 